

В. А. Быковский, А. В. Устинов, Статистика траекторий частиц в однородной задаче Синая для двумерной решетки, Функи. анализ и его прил., 2008, том 42, выпуск 3, 10-22

DOI: https://doi.org/10.4213/faa2909

Использование Общероссийского математического портала MathNet.Ru подразумевает, что вы прочитали и согласны с пользовательским соглашением

http://www . mathnet.ru/rus/agreement

Параметры загрузки:

IP : 18.234 .156 .22

26 апреля 2023 г., 16:14:48

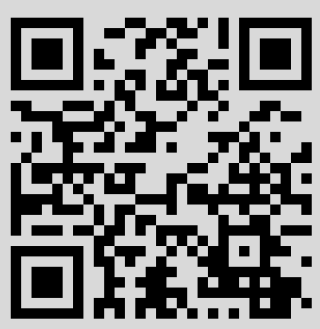


Функиионалъный анализ и его приложения

2008, т. 42, вып. 3, с. 10-22

УДК $511.336+511.9+517.987 .5$

\title{
Статистика траекторий частиц в однородной задаче Синая для двумерной решетки*
}

\author{
(c) 2008. В. А. Быковский, А. В. Устинов
}

\section{Обозначения}

1. $\|x\|-$ расстояние от вещественного числа $x$ до ближайшего целого числа.

2. $\varphi(d)$ - количество целых чисел от 1 до $d$, взаимно простых с $d$ (функция Эйлера).

3. $\mu(d)$ - функция Мебиуса.

4. Для натурального $q$ и целого $a$ через $\delta_{q}(a)$ будем обозначать характеристическую функцию делимости на $q$ :

$$
\delta_{q}(a)=\left\{\begin{array}{lll}
1, & \text { если } a \equiv 0 & (\bmod q), \\
0, & \text { если } a \not \equiv 0 & (\bmod q) .
\end{array}\right.
$$

5. Конечные разности функции $a(u, v)$ :

$$
\begin{gathered}
\Delta_{1,0} a(u, v)=a(u+1, v)-a(u, v), \quad \Delta_{0,1} a(u, v)=a(u, v+1)-a(u, v), \\
\Delta_{1,1} a(u, v)=\Delta_{0,1}\left(\Delta_{1,0} a(u, v)\right)=\Delta_{1,0}\left(\Delta_{0,1} a(u, v)\right) .
\end{gathered}
$$

\section{Введение}

Пусть $0<h<1 /(2 \sqrt{2})$ и $T>0$. Открытый круг радиуса $h$ с центром в некоторой точке назовем $h$-окрестностью этой точки. Определим $\Omega_{h}(T)$ как подмножество в $[0,2 \pi)$, состоящее из углов $\varphi$, для которых луч

$$
\{(t \cos \varphi, t \sin \varphi) \mid t \geqslant 0\}
$$

пересекает $h$-окрестность некоторой целочисленной точки $(m, n) \neq(0,0)$ из круга

$$
\left\{(x, y) \in \mathbb{R}^{2} \mid x^{2}+y^{2} \leqslant T^{2}\right\} .
$$

Обозначим через $G_{h}(T)$ нормированную меру множества $\Omega_{h}(T)$ :

$$
G_{h}(T)=\frac{1}{2 \pi} \operatorname{mes} \Omega_{h}(T) \in[0,1] .
$$

В 1918 г. Д. Пойа (см. [2, теория чисел, задача 239]) доказал, что $G_{h}(T)=1$ для всех $T \geqslant h^{-1}$. Отвечая на вопрос, поставленный в 1981 г. Я. Г. Синаем, в

* Исследования первого автора выполнены при поддержке фонда INTAS, грант 03-515070, фонда РФФИ, грант 07-01-00306, и проекта ДВО РАН 06-I-П-13-047. Исследования второго автора выполнены при поддержке фонда INTAS, грант 03-51-5070, фонда РФФИ, грант 07-01-00306, проекта ДВО РАН 06-III-А-01-017 и Фонда содействия отечественной науке. 
совместной работе [4] Ф. П. Бока, Р. Н. Гологан и А. Захареску доказали, что для любого $\varepsilon>0$ равномерно по $T \in\left[0, h^{-1}\right]$

$$
G_{h}(T)=\int_{0}^{h T} \sigma(t) d t+O_{\varepsilon}\left(h^{1 / 8-\varepsilon}\right)
$$

где

$$
\sigma(t)= \begin{cases}\frac{12}{\pi^{2}}, & \text { если } 0 \leqslant t \leqslant \frac{1}{2} \\ \frac{12}{\pi^{2}}\left(\frac{1}{t}-1\right)\left(1-\log \left(\frac{1}{t}-1\right)\right), & \text { если } \frac{1}{2}<t \leqslant 1 .\end{cases}
$$

С физической точки зрения величину $G_{h}(T)$ можно интерпретировать как функцию распределения длин свободного пробега частиц, движущихся прямолинейно из начала координат до первого попадания в $h$-окрестность некоторой ненулевой целочисленной точки. Речь идет об однородной двумерной модели «периодический газ Лоренца».

В связи с этим представляется интересной следующая более общая задача. Пусть $(m, n)=\left(m_{h}(\varphi), n_{h}(\varphi)\right) \in \mathbb{Z}^{2} \backslash\{(0,0)\}$ - центр первой $h$-окрестности, в которую попадет луч $(1)$. Обозначим через $T_{h}(\varphi)$ расстояние от начала координат до $M_{h}(\varphi)$ - проекции точки $\left(m_{h}(\varphi), n_{h}(\varphi)\right)$ на луч $(1)$. Введем также величину $U_{h}(\varphi) \in[-h, h]$, которая по абсолютной величине совпадает с расстоянием от $\left(m_{h}(\varphi), n_{h}(\varphi)\right)$ до $M_{h}(\varphi)$. При этом $U_{h}(\varphi)>0\left(U_{h}(\varphi)<0\right)$, если при движении частицы по лучу $(1)$ точка $\left(m_{h}(\varphi), n_{h}(\varphi)\right)$ остается справа (слева). Удобно рассматривать нормированные значения этих величин:

$$
t_{h}(\varphi)=h \cdot T_{h}(\varphi) \in[0,1], \quad u_{h}(\varphi)=h^{-1} \cdot U_{h}(\varphi) \in[-1,1] .
$$

Ориентируясь на терминологию из ядерной физики, назовем $u_{h}(\varphi)$ нормированным прицельным параметром; $t_{h}(\varphi)$ будем называть нормированным свободным пробегом.

Пусть $0 \leqslant t_{0} \leqslant 1$ и $-1 \leqslant u_{1} \leqslant u_{2} \leqslant 1$. Как обычно, $\chi_{I}(\ldots)$ - характеристическая функция промежутка $I$ на вещественной прямой. Главным результатом работы является следующее утверждение.

Теорема. При любом $\varepsilon>0$ для бункиии распределения

$$
\Phi(h)=\Phi\left(h ; \varphi_{0}, t_{0}, u_{1}, u_{2}\right)=\frac{1}{2 \pi} \int_{0}^{\varphi_{0}} \chi_{\left[0, t_{0}\right]}\left(t_{h}(\varphi)\right) \chi_{\left[u_{1}, u_{2}\right]}\left(u_{h}(\varphi)\right) d \varphi
$$

равномерно по $t_{0}, u_{1}, u_{2} u \varphi_{0} \in[0,2 \pi]$ справедлива асимптотическая формула

$$
\Phi(h)=\int_{0}^{\varphi_{0}} \int_{0}^{t_{0}} \int_{u_{1}}^{u_{2}} \rho(\varphi, t, u) d \varphi d t d u+O_{\varepsilon}\left(h^{1 / 2-\varepsilon}\right),
$$

əəe $h \rightarrow 0 u$

$$
\rho(\varphi, t, u)=\rho(t, u)= \begin{cases}\frac{3}{\pi^{3}}, & \text { если }|u| \leqslant \frac{1}{t}-1 \\ \frac{3}{\pi^{3}} \frac{1}{|u|}\left(\frac{1}{t}-1\right), & \text { если }|u|>\frac{1}{t}-1\end{cases}
$$

Замечание 1. Если положить $\varphi_{0}=2 \pi$, а также $u_{1}=-1$ и $u_{2}=1$, то получим уточнение остаточного члена в асимптотической формуле (2), доказанной в работе [4]. 
Замечание 2. С физической точки зрения функцию $\rho(\varphi, t, u)$ можно интерпретировать как плотность частиц, движущихся прямолинейно из начала координат под углом $\varphi$ к оси абсцисс, которые проходят до первого рассеяния в $h$-окрестностях целочисленных точек расстояние $T=h^{-1} \cdot t$ (свободный пробег) и попадают в $h$-окрестности с прицельным параметром $U=h \cdot u$.

Замечание 3. Плотность распределения $\rho(\varphi, t, u)$ не зависит от угла $\varphi$ (изотропность).

\section{§1. Применение непрерывных дробей}

Очевидные симметрии в рассматриваемой задаче приводят к равенствам

$$
t_{h}(\varphi)=t_{h}(-\varphi)=t_{h}(\varphi+\pi / 2), \quad u_{h}(\varphi)=-u_{h}(-\varphi)=u_{h}(\varphi+\pi / 2) .
$$

Поэтому теорему достаточно доказать только для $\varphi_{0} \in[0, \pi / 4]$, что и будет предполагаться в дальнейшем. При этом $\alpha=\operatorname{arctg} \varphi \in[0,1]$.

Положим

$$
\begin{aligned}
& l_{\varphi}(x, y)=x \sin \varphi-y \cos \varphi=\frac{\alpha x-y}{\sqrt{1+\alpha^{2}}}, \\
& l_{\varphi}^{*}(x, y)=x \cos \varphi+y \sin \varphi=\frac{x+\alpha y}{\sqrt{1+\alpha^{2}}} .
\end{aligned}
$$

Замечание 4. Из определений величин $l_{\varphi}(x, y)$ и $l_{\varphi}^{*}(x, y)$ немедленно следует, что $\left(m_{h}(\varphi), n_{h}(\varphi)\right)$ - целочисленная точка $(m, n)$ с $\left|l_{\varphi}(m, n)\right|<h, m>0$ и $n \geqslant 0$, для которой величина $l_{\varphi}^{*}(m, n)$ принимает наименьшее значение, равное

$$
h^{-1} \cdot t_{0}(\varphi)=l_{\varphi}^{*}\left(m_{h}(\varphi), n_{h}(\varphi)\right) .
$$

При этом

$$
h \cdot u_{0}(\varphi)=l_{\varphi}\left(m_{h}(\varphi), n_{h}(\varphi)\right) .
$$

Лемма 1. Целочисленная пара $\left(m_{h}(\varphi), n_{h}(\varphi)\right)$ однозначно определяется из условий

$$
m_{h}(\varphi)=\min \left\{m \in \mathbb{N} \mid\|\alpha m\|<h \sqrt{1+\alpha^{2}}\right\}, \quad\left|\alpha m_{h}(\varphi)-n_{h}(\varphi)\right|<\frac{1}{2} .
$$

Доказательство. Предположим, что для некоторого натурального $m$, меньшего $m_{h}(\varphi)$, выполняется неравенство

$$
\|\alpha m\| \leqslant\left\|\alpha m_{h}(\varphi)\right\| .
$$

Тогда при некотором целом $n \geqslant 0$

$$
|\alpha m-n|=\|\alpha m\| \leqslant\left\|\alpha m_{h}(\varphi)\right\|=\left|\alpha m_{h}(\varphi)-n_{h}(\varphi)\right|<h \sqrt{1+\alpha^{2}} \leqslant \frac{1}{2 \sqrt{2}} \sqrt{2}=\frac{1}{2} .
$$

Отсюда следует, что

$$
\begin{aligned}
n<\alpha m+\frac{1}{2} & \leqslant \alpha m_{h}(\varphi)+\frac{1}{2}=\alpha m_{h}(\varphi)-n_{h}(\varphi)+n_{h}(\varphi)+\frac{1}{2} \\
& <n_{h}(\varphi)+h \sqrt{1+\alpha^{2}}+\frac{1}{2}<n_{h}(\varphi)+1 .
\end{aligned}
$$

То есть $n \leqslant n_{h}(\varphi)$. Но тогда

$m \cos \varphi+n \sin \varphi<m_{h}(\varphi) \cos \varphi+n_{h}(\varphi) \sin \varphi$. 
Это неравенство противоречит замечанию 4. Значит, наше предположение неверно и лемма 1 полностью доказана.

Напомним, что любое вещественное число $x$ каноническим способом разлагается в непрерывную дробь,

$$
x=\left[q_{0} ; q_{1}, \ldots, q_{i}, \ldots\right]=q_{0}+\frac{1}{q_{1}+\ddots \cdot+\frac{1}{q_{i}+\cdot}},
$$

с целой частью $q_{0}=[x]$ и неполными частными $q_{i}=q_{i}(x) \in \mathbb{N}$ при $i \geqslant 1$. Она конечна только для рациональных $x$, и в этом случае ее последнее неполное частное (если оно присутствует в разложении) больше 1. По определению

$$
P_{i}=P_{i}(x) \quad \text { и } \quad Q_{i}=Q_{i}(x) \quad(i=1,2, \ldots)
$$

- числитель (целое число) и знаменатель (натуральное число) несократимой $i$-й подходящей дроби

$$
\frac{P_{i}}{Q_{i}}=\left[q_{0} ; q_{1}, \ldots, q_{i-1}\right]
$$

для $x$. При этом $P_{1}=q_{0}$ и $Q_{1}=1$.

Лемма 2. Целые $n_{h}(\varphi)$ и $m_{h}(\varphi)$ - числитель и знаменатель некоторой подходящей дроби для $\alpha=\operatorname{tg} \varphi \in[0,1]$.

Доказательство. Из леммы 1 следует, что не существует натуральных $m$, меньших $m_{h}(\varphi)$, для которых $\|\alpha m\| \leqslant\left\|\alpha m_{h}(\varphi)\right\|$. Отсюда, воспользовавшись теоремой Лагранжа о наилучших приближениях (см. [3]), получаем утверждение леммы 2.

Обозначим через $\mathscr{M}$ множество всех целочисленных матриц

$$
S=\left(\begin{array}{ll}
P & P^{\prime} \\
Q & Q^{\prime}
\end{array}\right)=\left(\begin{array}{ll}
P(S) & P^{\prime}(S) \\
Q(S) & Q^{\prime}(S)
\end{array}\right)
$$

с определителем $\operatorname{det} S= \pm 1$, у которых

$$
1 \leqslant Q \leqslant Q^{\prime}, \quad 0 \leqslant P \leqslant Q, \quad 1 \leqslant P^{\prime} \leqslant Q^{\prime} .
$$

Оно разбивается на два непересекающихся подмножества $\mathscr{M}_{+}$и $\mathscr{M}_{-}$, состоящих из матриц с определителями +1 и -1 соответственно. В дальнейшем будем предполагать, что * есть либо + , либо -, либо пустой символ. В зависимости от контекста, если это не приводит к недоразумениям, мы будем использовать обозначения $P, P^{\prime}, Q, Q^{\prime}$ вместо $P(S), P^{\prime}(S), Q(S), Q^{\prime}(S)$ для элементов матрицы $S$ в соответствии с $(5)$.

Пусть $X \geqslant 1$ и

$$
\mathscr{M}_{*}(X)=\left\{S \in \mathscr{M}_{*} \mid\left(P^{\prime}\right)^{2}+\left(Q^{\prime}\right)^{2} \leqslant X^{2}\right\} .
$$

Для $\alpha \in(0,1)$ матрица

$$
S_{i}(\alpha)=\left(\begin{array}{cc}
P_{i}(\alpha) & P_{i+1}(\alpha) \\
Q_{i}(\alpha) & Q_{i+1}(\alpha)
\end{array}\right)
$$


есть элемент из $\mathscr{M}$ с определителем $\operatorname{det} S_{i}(\alpha)=(-1)^{i}$. Обозначим через $\widetilde{\mathbb{N}}$ множество всех непустых конечных наборов $\left(q_{1}, \ldots, q_{n}\right)$, составленных из натуральных чисел. Построим отображение

$$
\mathscr{B}: \quad \widetilde{\mathbb{N}}=\bigcup_{k=1}^{\infty} \mathbb{N}^{k} \rightarrow \mathscr{M},
$$

положив

$$
\mathscr{B}\left(q_{1}, \ldots, q_{n}\right)=S=S\left(q_{1}, \ldots, q_{n}\right)=\left(\begin{array}{cc}
0 & 1 \\
1 & q_{1}
\end{array}\right) \cdots\left(\begin{array}{cc}
0 & 1 \\
1 & q_{n}
\end{array}\right) .
$$

При этом $S$ имеет вид (5) с

$$
\frac{P}{Q}=\left[0 ; q_{1}, \ldots, q_{n-1}\right] \quad \text { и } \quad \frac{P^{\prime}}{Q^{\prime}}=\left[0 ; q_{1}, \ldots, q_{n}\right] .
$$

С помощью свойств непрерывных дробей легко проверяется следующее утверждение.

Лемма 3. Отображсение $\mathscr{B}$ - биекиия.

Любопытно отметить (хотя это и не понадобится нам в дальнейшем), что $\mathscr{M}$ - полугруппа относительно обычного умножения матриц, а биекция $\mathscr{B}$ на самом деле есть полугрупповой изоморфизм. При этом

$$
\left(q_{1}, \ldots, q_{n}\right) *\left(q_{n+1}, \ldots, q_{n+m}\right)=\left(q_{1}, \ldots, q_{n}, q_{n+1}, \ldots, q_{n+m}\right)
$$

- соответствующая операция на конечных наборах натуральных чисел.

Сопоставим каждой матрице

$$
S=S\left(q_{1}, \ldots, q_{n}\right)=\left(\begin{array}{ll}
P & P^{\prime} \\
Q & Q^{\prime}
\end{array}\right) \in \mathscr{M}
$$

полуинтервал

$$
\begin{aligned}
I(S) & =\left\{\alpha=\left[0 ; q_{1}, \ldots, q_{n-1}, q_{n}+\beta\right] \mid 0 \leqslant \beta<1\right\}= \\
& =\left\{\alpha=\frac{P^{\prime}+\beta P}{Q^{\prime}+\beta Q} \mid 0 \leqslant \beta<1\right\}=\left\{\alpha \in(0,1] \mid-1<\frac{\alpha Q^{\prime}-P^{\prime}}{\alpha Q-P} \leqslant 0\right\}
\end{aligned}
$$

При этом в случае $S \in \mathscr{M}_{+}$

$$
I(S)=\left[\frac{P^{\prime}}{Q^{\prime}}, \frac{P^{\prime}+P}{Q^{\prime}+Q}\right)=\left[\frac{P^{\prime}}{Q^{\prime}}, \frac{P^{\prime}}{Q^{\prime}}+\frac{1}{Q^{\prime}\left(Q^{\prime}+Q\right)}\right),
$$

а в случае $S \in \mathscr{M}_{-}$

$$
I(S)=\left(\frac{P^{\prime}+P}{Q^{\prime}+Q}, \frac{P^{\prime}}{Q^{\prime}}\right]=\left(\frac{P^{\prime}}{Q^{\prime}}-\frac{1}{Q^{\prime}\left(Q^{\prime}+Q\right)}, \frac{P^{\prime}}{Q^{\prime}}\right] .
$$

Далее, положим для $S \in \mathscr{M}_{+}$

$$
I_{h}(S)=\left\{\alpha \in(0,1] \mid 0 \leqslant \alpha Q^{\prime}-P^{\prime}<h \sqrt{1+\alpha^{2}} \leqslant-\alpha Q+P\right\},
$$

а для $S \in \mathscr{M}$

$$
I_{h}(S)=\left\{\alpha \in(0,1] \mid 0 \leqslant-\alpha Q^{\prime}+P^{\prime}<h \sqrt{1+\alpha^{2}} \leqslant \alpha Q-P\right\} .
$$

Из последнего представления для $I(S)$ в $(6)$ немедленно вытекает включение

$$
I_{h}(S) \subset I(S),
$$


а также следующее утверждение.

Замечание 5. Величины $P / Q$ и $P^{\prime} / Q^{\prime}$ - последовательные подходящие дроби для $\alpha$, однозначно определяемые по $\alpha$ и $h$.

Пусть

$$
f_{S}(\beta)=Q^{\prime} \beta-h \sqrt{1+\left(\frac{P^{\prime}}{Q^{\prime}}+\operatorname{det} S \cdot \beta\right)^{2}} .
$$

Так как

$$
f_{S}^{\prime}(\beta)=Q^{\prime}-h \frac{\operatorname{det} S \cdot\left(P^{\prime} / Q^{\prime}\right)+\beta}{\sqrt{1+\left(\operatorname{det} S \cdot\left(P^{\prime} / Q^{\prime}\right)+\beta\right)^{2}}}>Q^{\prime}-h>0
$$

и

$$
f_{S}(0)=-h \sqrt{1+\left(\frac{P^{\prime}}{Q^{\prime}}\right)^{2}}<0
$$

то уравнение $f_{S}(\beta)=0$ имеет единственный положительный корень, который мы обозначим через $\lambda=\lambda_{S}(h)$. Заметим, что

$$
\left(\frac{Q^{\prime} \lambda}{h}\right)^{2}=1+\left(\frac{P^{\prime}}{Q^{\prime}}+\operatorname{det} S \cdot \lambda\right)^{2} \leqslant 3+2 \lambda^{2}
$$

TaK каK

$$
2 \leqslant \frac{1}{4}\left(\frac{1}{h}\right)^{2} \leqslant \frac{1}{4}\left(\frac{Q^{\prime}}{h}\right)^{2}
$$

TO

$$
\lambda^{2} \leqslant \frac{3}{\left(Q^{\prime} / h\right)^{2}-2} \leqslant \frac{3}{\left(Q^{\prime} / h\right)^{2}-\frac{1}{4}\left(Q^{\prime} / h\right)^{2}}=\left(\frac{2 h}{Q^{\prime}}\right)^{2} .
$$

Поэтому

$$
\lambda_{S}(h) \leqslant \frac{2 h}{Q^{\prime}}
$$

Теперь рассмотрим функцию

$$
g_{S}(\beta)=Q \beta+h \sqrt{1+\left(\frac{P^{\prime}}{Q^{\prime}}+\operatorname{det} S \cdot \beta\right)^{2}},
$$

для которой

$$
g_{S}^{\prime}(\beta)=Q+h \frac{\operatorname{det} S \cdot\left(P^{\prime} / Q^{\prime}\right)+\beta}{\sqrt{1+\left(\operatorname{det} S \cdot\left(P^{\prime} / Q^{\prime}\right)+\beta\right)^{2}}}>Q-h>0 .
$$

Это возрастающая функция и

$$
g_{S}(0)=h \sqrt{1+\left(\frac{P^{\prime}}{Q^{\prime}}\right)^{2}} .
$$

Поэтому справедливо следующее утверждение.

Замечание 6. Уравнение $g_{S}(\beta)=1 / Q^{\prime}$ имеет единственный (неотрицательный) корень $\eta=\eta_{S}(h)$ только в случае, когда

$$
h \sqrt{1+\left(\frac{P^{\prime}}{Q^{\prime}}\right)^{2}} \leqslant \frac{1}{Q^{\prime}}, \quad \text { т. e. } S \in \mathscr{M}\left(h^{-1}\right) .
$$


Подводя итог сказанному выше и обращаясь к определению множества $I_{h}(S)$, приходим к следующему утверждению.

Замечание 7. Множество $I_{h}(S)$ непусто только для $S \in \mathscr{M}\left(h^{-1}\right)$, и при $S \in \mathscr{M}_{+}\left(h^{-1}\right)$ оно совпадает с

$$
\left[\frac{P^{\prime}}{Q^{\prime}}, \frac{P^{\prime}}{Q^{\prime}}+\lambda_{S}(h)\right) \cap\left[\frac{P^{\prime}}{Q^{\prime}}, \frac{P^{\prime}}{Q^{\prime}}+\eta_{S}(h)\right],
$$

а при $S \in \mathscr{M}_{-}\left(h^{-1}\right)-\mathrm{c}$

$$
\left(\frac{P^{\prime}}{Q^{\prime}}-\lambda_{S}(h), \frac{P^{\prime}}{Q^{\prime}}\right] \cap\left[\frac{P^{\prime}}{Q^{\prime}}-\eta_{S}(h), \frac{P^{\prime}}{Q^{\prime}}\right] .
$$

Лемма 4. Множества $I_{h}(S)$ попарно не пересекаются и

$$
\bigcup_{S \in \mathscr{M}\left(h^{-1}\right)} I_{h}(S)=\left[\frac{h}{\sqrt{1-h^{2}}}, 1\right] .
$$

Доказательство. Первая часть утверждения леммы непосредственно следует из замечания 5. Далее, пусть $P_{j}=P_{j}(\alpha)$ и $Q_{j}=Q_{j}(\alpha)$ - числитель и знаменатель $j$-й подходящей дроби для числа $\alpha \in(0,1]$. Так как последовательность

$$
\alpha=\alpha_{1}=\alpha Q_{1}-P_{1}, \quad \ldots, \quad \alpha_{j}=\alpha Q_{j}-P_{j}, \quad \ldots
$$

по абсолютной величине монотонно убывает к нулю, то существует

$$
i=i_{h}(\alpha)=\min \left\{j|| \alpha Q_{j}-P_{j} \mid<h \sqrt{1+\alpha^{2}}\right\} .
$$

Если $i=1$, то (напомним, что $P_{1}=0$ и $Q_{1}=1$ )

$$
\left|\alpha Q_{1}-P_{1}\right|=\alpha<h \sqrt{1+\alpha^{2}}, \quad \text { т. е. } \alpha \in\left(0, \frac{h}{\sqrt{1-h^{2}}}\right) .
$$

Для всех остальных $\alpha$ всегда $i \geqslant 2$ и при этом $\left|\alpha Q_{i-1}-P_{i-1}\right| \geqslant h \sqrt{1+\alpha^{2}}$. Принимая во внимание знакопеременность последовательности (9) и замечание 6 , а также положив

$$
S=\left(\begin{array}{ll}
P_{i-1} & P_{i} \\
Q_{i-1} & Q_{i}
\end{array}\right)
$$

получим вторую часть утверждения леммы 4.

\section{§2. Вспомогательные преобразования}

В соответствии с введенными ранее обозначениями положим

$$
\xi_{S}(\alpha)=\chi_{\left[u_{1}, u_{2}\right]}\left(\frac{\alpha Q^{\prime}-P^{\prime}}{h \sqrt{1+\alpha^{2}}}\right), \quad I_{h}^{\left(\alpha_{0}\right)}(S)=\left[0, \alpha_{0}\right] \cap I_{h}(S) .
$$

Переходя к переменной $\alpha=\operatorname{tg} \varphi \in[0,1]$ в интеграле, определяющем $\Phi(h)$ (см. формулировку теоремы во введении), и применяя лемму 4, получаем

$$
\Phi(h)=\frac{1}{2 \pi} \sum_{S \in \mathscr{M}\left(h^{-1}\right)} \int_{I_{h}^{\left(\alpha_{0}\right)}(S)} \chi_{\left[0, t_{0}\right]}\left(\frac{h\left(Q^{\prime}+\alpha P^{\prime}\right)}{\sqrt{1+\alpha^{2}}}\right) \xi_{S}(\alpha) \frac{d \alpha}{1+\alpha^{2}}+O(h) .
$$


Taк как

$$
\left(\frac{Q^{\prime}+\alpha P^{\prime}}{\sqrt{1+\alpha^{2}}}\right)^{2}+\left(\frac{\alpha Q^{\prime}-P^{\prime}}{\sqrt{1+\alpha^{2}}}\right)^{2}=\left(P^{\prime}\right)^{2}+\left(Q^{\prime}\right)^{2},
$$

то для любого $\alpha \in I_{h}(S)$

$$
\left(P^{\prime}\right)^{2}+\left(Q^{\prime}\right)^{2}-h^{2}<\left(\frac{Q^{\prime}+\alpha P^{\prime}}{\sqrt{1+\alpha^{2}}}\right)^{2} \leqslant\left(P^{\prime}\right)^{2}+\left(Q^{\prime}\right)^{2} .
$$

Поэтому если

$$
\left(t_{0} h^{-1}\right)^{2}<\left(P^{\prime}\right)^{2}+\left(Q^{\prime}\right)^{2}
$$

и для некоторого $\alpha \in I_{h}(S)$

$$
\chi_{\left[0, t_{0}\right]}\left(\frac{h\left(Q^{\prime}+\alpha P^{\prime}\right)}{\sqrt{1+\alpha^{2}}}\right)=1,
$$

TO

$$
\left(t_{0} h^{-1}\right)^{2}<\left(P^{\prime}\right)^{2}+\left(Q^{\prime}\right)^{2}<\left(t_{0} h^{-1}\right)^{2}+h^{2} \leqslant\left(t_{0} h^{-1}\right)^{2}+1 / 8 .
$$

Отсюда, воспользовавшись оценкой

$$
\int_{I_{h}^{\left(\alpha_{0}\right)}(S)} d \alpha \leqslant \int_{I_{h}(S)} d \alpha \leqslant \lambda_{S}(h) \leqslant \frac{2 h}{Q^{\prime}}
$$

(см. также (8)), получаем равенство

$$
\Phi(h)=\frac{1}{2 \pi} \sum_{S \in \mathscr{M}\left(t_{0} h^{-1}\right)} \int_{I_{h}^{\left(\alpha_{0}\right)}(S)} \xi_{S}(\alpha) \frac{d \alpha}{1+\alpha^{2}}+R_{1},
$$

где

$$
R_{1} \ll h+\sum_{\substack{\left(t_{0} / h\right)^{2} \leqslant\left(P^{\prime}\right)^{2}+\left(Q^{\prime}\right)^{2}<\left(t_{0} / h\right)^{2}+1 / 8 \\ 1 \leqslant P^{\prime} \leqslant Q^{\prime}}} \frac{h}{Q^{\prime}} \ll h+\frac{h}{t_{0} / h+1 / 2} \sum_{Q^{\prime} \leqslant t_{0} / h+1 / 2} 1 \ll h .
$$

В соответствии с оценкой (8) для $\alpha \in I_{h}(S)$ по теореме Лагранжа

$$
\left|\frac{1}{1+\alpha^{2}}-\frac{1}{1+\left(P^{\prime} / Q^{\prime}\right)^{2}}\right| \leqslant\left|\alpha-\frac{P^{\prime}}{Q^{\prime}}\right| \leqslant \lambda_{S}(h) \leqslant \frac{2 h}{Q^{\prime}} .
$$

Следовательно,

$$
\Phi(h)=\frac{1}{2 \pi} \sum_{S \in \mathscr{M}\left(t_{0} h^{-1}\right)} \frac{1}{1+\left(P^{\prime} / Q^{\prime}\right)^{2}} \int_{I_{h}^{\left(\alpha_{0}\right)}(S)} \xi_{S}(\alpha) d \alpha+R_{2},
$$

где

$$
R_{2} \ll h+\sum_{1 \leqslant P^{\prime} \leqslant Q^{\prime} \leqslant h^{-1}} \frac{h}{Q^{\prime}} \cdot \frac{h}{Q^{\prime}} \ll h .
$$

Опираясь на неравенство

$$
\left|\alpha-\frac{P^{\prime}}{Q^{\prime}}\right| \leqslant \frac{2 h}{Q^{\prime}} \quad \forall \alpha \in I_{h}(S)
$$

и положив

$$
\mathscr{M}_{*}^{\left(\alpha_{0}\right)}(X)=\left\{S \in \mathscr{M}_{*}(X) \mid P^{\prime} \leqslant \alpha_{0} Q^{\prime}\right\}
$$


получим, что

$$
\Phi(h)=\frac{1}{2 \pi} \sum_{S \in \mathscr{M}^{\left(\alpha_{0}\right)}\left(t_{0} h^{-1}\right)} \frac{1}{1+\left(P^{\prime} / Q^{\prime}\right)^{2}} \int_{I_{h}(S)} \xi_{S}(\alpha) d \alpha+R_{3},
$$

где

$$
R_{3} \ll h+\sum_{\substack{1 \leqslant P^{\prime} \leqslant Q^{\prime} \leqslant h^{-1} \\\left|\alpha_{0} Q^{\prime}-P^{\prime}\right| \leqslant 2 h}} \frac{h}{Q^{\prime}} \ll h+h \sum_{Q^{\prime} \leqslant h^{-1}} \frac{1}{Q^{\prime}} \ll h \cdot \log \frac{1}{h} .
$$

Заметим что

1) для $0 \leqslant u_{1} \leqslant u_{2} \leqslant 1$

$$
\Phi\left(\ldots, u_{1}, u_{2}\right)=\Phi\left(\ldots, 0, u_{2}\right)-\Phi\left(\ldots, 0, u_{1}\right) ;
$$

2 длля $-1 \leqslant u_{1} \leqslant 0 \leqslant u_{2} \leqslant 1$

$$
\Phi\left(\ldots, u_{1}, u_{2}\right)=\Phi\left(\ldots, u_{1}, 0\right)+\Phi\left(\ldots, 0, u_{2}\right) ;
$$

3) для $-1 \leqslant u_{1} \leqslant u_{2} \leqslant 0$

$$
\Phi\left(\ldots, u_{1}, u_{2}\right)=\Phi\left(\ldots, u_{1}, 0\right)-\Phi\left(\ldots, u_{2}, 0\right) .
$$

Поэтому теорему достаточно доказать только в случаях $u_{1}=0$ и $u_{2}=u_{0}$ или $u_{1}=-u_{0}$ и $u_{2}=0$ с $u_{0} \in[0,1]$. При этом в соответствии с равенством $(10)$

$$
\Phi_{+}(h)=\Phi\left(h ; \varphi_{0}, t_{0}, 0, u_{0}\right)=\widetilde{\Phi}_{+}(h)+O\left(h \log \frac{1}{h}\right)
$$

и

$$
\Phi_{-}(h)=\Phi\left(h ; \varphi_{0}, t_{0},-u_{0}, 0\right)=\widetilde{\Phi}_{-}(h)+O\left(h \log \frac{1}{h}\right)
$$

где

$$
\widetilde{\Phi}_{ \pm}(h)=\frac{1}{2 \pi} \sum_{S \in \mathscr{M}_{ \pm}^{\left(\alpha_{0}\right)}\left(t_{0} h^{-1}\right)} \frac{\min \left\{\lambda_{S}\left(u_{0} h\right), \eta_{S}(h)\right\}}{1+\left(P^{\prime} / Q^{\prime}\right)^{2}} .
$$

Из уравнения, определяющего $\lambda_{S}(h)$, и оценки (8) следует, что

$$
\lambda_{S}(h)=\widetilde{\lambda}_{S}(h)+O\left(\left(\frac{h}{Q^{\prime}}\right)^{2}\right) \quad \text { с } \quad \widetilde{\lambda}_{S}(h)=\frac{h}{Q^{\prime}} \sqrt{1+\left(\frac{P^{\prime}}{Q^{\prime}}\right)^{2}} .
$$

Аналогично, для $\eta_{S}(h) \leqslant \lambda_{S}(h)$ выполняется асимптотическое равенство

$$
\eta_{S}(h)=\widetilde{\eta}_{S}(h)+O\left(\frac{h^{2}}{Q Q^{\prime}}\right) \quad \text { с } \quad \tilde{\eta}_{S}(h)=\frac{1}{Q Q^{\prime}}-\frac{h}{Q} \sqrt{1+\left(\frac{P^{\prime}}{Q^{\prime}}\right)^{2}} \text {. }
$$

Поэтому

$$
\min \left\{\lambda_{S}\left(u_{0} h\right), \eta_{S}(h)\right\}=\min \left\{\widetilde{\lambda}_{S}\left(u_{0} h\right), \widetilde{\eta}_{S}(h)\right\}+O\left(\frac{h^{2}}{Q Q^{\prime}}\right) .
$$

Положив

$$
\Psi(x, y)=\Psi\left(Q^{\prime} ; x, y\right)=\frac{\min \left\{u_{0} h Q^{\prime} \sqrt{1+x^{2}}, \frac{1}{y}\left(1-h Q^{\prime} \sqrt{1+x^{2}}\right)\right\}}{1+x^{2}},
$$


окончательно получаем, что

$$
\widetilde{\Phi}_{ \pm}(h)=\frac{1}{2 \pi} \sum_{S \in \mathscr{M}_{ \pm}^{\left(\alpha_{0}\right)}\left(t_{0} h^{-1}\right)} \frac{1}{\left(Q^{\prime}\right)^{2}} \Psi\left(\frac{P^{\prime}}{Q^{\prime}}, \frac{Q}{Q^{\prime}}\right)+R_{4}
$$

где

$$
R_{4} \ll \sum_{1 \leqslant Q \leqslant Q^{\prime} \leqslant h^{-1}} \frac{h^{2}}{Q Q^{\prime}} \ll h^{2} \log ^{2} \frac{1}{h} \ll h .
$$

Введем функцию

$$
\Psi_{0}(x, y)=\Psi_{0}\left(Q^{\prime} ; x, y\right)= \begin{cases}\Psi\left(Q^{\prime} ; x, y\right), & \text { если } x \leqslant \alpha_{0}, \sqrt{1+x^{2}} \leqslant t_{0}\left(h Q^{\prime}\right)^{-1}, \\ 0 & \text { в остальных случаях. }\end{cases}
$$

Для фиксированного значения $Q^{\prime}=q$ числа $P^{\prime}$ и $Q$ должны быть решениями сравнения $a b \equiv \pm 1(\bmod q)$. Поэтому величины $\widetilde{\Phi}_{ \pm}(h)$ могут быть переписаны в виде

$$
\widetilde{\Phi}_{ \pm}(h)=\frac{1}{2 \pi} \sum_{q \leqslant h^{-1}} \frac{1}{q^{2}} \sum_{a, b=1}^{q} \delta_{q}(a b \pm 1) \Psi_{0}\left(\frac{a}{q}, \frac{b}{q}\right)+O(h)=\frac{1}{2 \pi} \sum_{q \leqslant h^{-1}} \frac{1}{q^{2}} W_{ \pm}(q)+O(h),
$$

где

$$
W_{ \pm}(q)=\sum_{a, b=1}^{q} \delta_{q}(a b \pm 1) \Psi_{0}\left(\frac{a}{q}, \frac{b}{q}\right)
$$

\section{§3. Применение оценок сумм Клостермана}

Далее предполагается, что $\varepsilon-$ сколь угодно малая положительная константа. С помощью оценок сумм Клостермана, принадлежащих Өстерману [5], стандартным образом доказывается следующее утверждение.

Лемма 5. Пусть $q, k, l$ - натуральные числа. Тогда

$$
\sum_{a=1}^{k} \sum_{b=1}^{l} \delta_{q}(a b \pm 1)=\frac{\varphi(q)}{q^{2}} k l+O_{\varepsilon}\left(q^{1 / 2+\varepsilon}\right) .
$$

Доказательство см. в [1].

Лемма 6. Пусть $q$ - натуральное число, $q \ll h^{-1}$. Тогда для суммъ $W_{ \pm}(q)$, заданной равенством (15), справедлива асимптотическая формула

$$
W_{ \pm}(q)=\varphi(q) \int_{0}^{1} \int_{0}^{1} \Psi_{0}(q ; x, y) d x d y+O_{\varepsilon}\left(h q^{3 / 2+\varepsilon}\right) .
$$

Доказательство. Применив к сумме $W_{ \pm}(q)$ преобразование Абеля

$$
\sum_{n=1}^{q} f(n) g(n)=g(q+1) \sum_{n=1}^{q} f(n)-\sum_{k=1}^{q}\left(\sum_{n=1}^{k} f(n)\right)(g(k+1)-g(k))
$$


последовательно по переменным $a$ и $b$ (сначала выбираем $f(a)=\delta_{q}(a b-1)$, $g(a)=\Psi_{0}(a / q, b / q)$, а затем $\left.f(b)=\sum_{a=1}^{k} \delta_{q}(a b-1), g(b)=\Delta_{1,0} \Psi_{0}(a / q, b / q)\right)$, находим, что

$$
W_{ \pm}(q)=\sum_{k, l=1}^{q} \Delta_{1,1} \Psi_{0}\left(\frac{k}{q}, \frac{l}{q}\right) \sum_{a=1}^{k} \sum_{b=1}^{l} \delta_{q}(a b-1) .
$$

Применяя к двойной внутренней сумме лемму 5, получаем

$$
\begin{aligned}
W_{ \pm}(q) & =\frac{\varphi(q)}{q^{2}} \sum_{k, l=1}^{q} \Delta_{1,1} \Psi_{0}\left(\frac{k}{q}, \frac{l}{q}\right) k l+O_{\varepsilon}\left(A q^{1 / 2+\varepsilon}\right) \\
& =\frac{\varphi(q)}{q^{2}} \sum_{k, l=1}^{q} \Delta_{1,1} \Psi_{0}\left(\frac{k}{q}, \frac{l}{q}\right) \sum_{u=1}^{k} \sum_{v=1}^{l} 1+O_{\varepsilon}\left(A q^{1 / 2+\varepsilon}\right),
\end{aligned}
$$

где

$$
A=\sum_{k, l=1}^{q}\left|\Delta_{1,1} \Psi_{0}\left(\frac{k}{q}, \frac{l}{q}\right)\right|
$$

Делая суммирование по $a$ и $b$ внешним, а потом суммируя по $k$ и $l$, находим

$$
\begin{aligned}
W_{ \pm}(q) & =\frac{\varphi(q)}{q^{2}} \sum_{a, b=1}^{q} \Psi_{0}\left(\frac{a}{q}, \frac{b}{q}\right)+O_{\varepsilon}\left(A q^{1 / 2+\varepsilon}\right) \\
& =\varphi(q) \int_{0}^{1} \int_{0}^{1} \Psi_{0}(x, y) d x d y+O_{\varepsilon}\left(A q^{1 / 2+\varepsilon}+h q\right) .
\end{aligned}
$$

Из определения функции $\Psi_{0}(x, y)$ немедленно следует, что

$$
\Delta_{1,1} \Psi_{0}\left(\frac{k}{q}, \frac{l}{q}\right) \geqslant 0
$$

во всех точках $(k / q, l / q)$ за исключением, быть может, тех, для которых кривая

$$
u_{0} h q \sqrt{1+x^{2}}=\frac{1}{y}\left(1-h q \sqrt{1+x^{2}}\right) \quad(0 \leqslant x, y \leqslant 1)
$$

пересекает квадрат $[k / q,(k+1) / q] \times[l / q,(l+1) / q]$. Таких точек $O(q)$, и в каждой из них

$$
\Delta_{1,1} \Psi_{0}\left(\frac{k}{q}, \frac{l}{q}\right) \ll \Delta_{1,0} \Psi_{0}\left(\frac{k}{q}, \frac{l}{q}\right) \ll h .
$$

Поэтому

$$
\begin{aligned}
A & =\sum_{k, l=1}^{q} \Delta_{1,1} \Psi_{0}\left(\frac{k}{q}, \frac{l}{q}\right)+O(h q) \\
& =\Psi_{0}(0,0)-\Psi_{0}\left(\frac{q+1}{q}, 0\right)-\Psi_{0}\left(0, \frac{q+1}{q}\right)+\Psi_{0}\left(\frac{q+1}{q}, \frac{q+1}{q}\right)+O(h q) \\
& =O(h q)
\end{aligned}
$$


и

$$
W_{ \pm}(q)=\varphi(q) \int_{0}^{1} \int_{0}^{1} \Psi_{0}(q ; x, y) d x d y+O_{\varepsilon}\left(h q^{3 / 2+\varepsilon}\right) .
$$

Теперь у нас есть все необходимое для доказательства основного результата. С учетом формул (11) и (12) достаточно проверить, что

$$
\widetilde{\Phi}_{ \pm}(h)=\int_{0}^{\alpha_{0}} \frac{d x}{1+x^{2}} \int_{0}^{t_{0}} d r \int_{0}^{u_{0}} \rho(r, u) d u+O_{\varepsilon}\left(h^{1 / 2-\varepsilon}\right),
$$

где $\rho(r, u)$ определено в формулировке теоремы.

Подставляя результат леммы 6 в формулу (14), получаем

$$
\begin{aligned}
\widetilde{\Phi}_{ \pm}(h) & =\frac{1}{2 \pi} \sum_{q \leqslant h^{-1}} \frac{\varphi(q)}{q^{2}} \int_{0}^{1} \int_{0}^{1} \Psi_{0}(q ; x, y) d x d y+O_{\varepsilon}\left(h^{1 / 2-\varepsilon}\right) \\
& =\frac{1}{2 \pi} \sum_{d \leqslant h^{-1}} \frac{\mu(d)}{d^{2}} \sum_{n \leqslant(d h)^{-1}} \int_{0}^{1} \int_{0}^{1} \frac{\Psi_{0}(n d ; x, y)}{n} d x d y+O_{\varepsilon}\left(h^{1 / 2-\varepsilon}\right) .
\end{aligned}
$$

Преобразуем внутреннюю сумму, пользуясь определением функции $\Psi_{0}(x, y)$ :

$$
\begin{aligned}
& \sum_{n \leqslant(d h)^{-1}} \int_{0}^{1} \int_{0}^{1} \frac{\Psi_{0}(n d ; x, y)}{n} d x d y \\
& \quad=d h \int_{0}^{\alpha_{0}} \frac{d x}{1+x^{2}} \int_{0}^{1} d y \sum_{n \leqslant\left(d h \sqrt{1+x^{2}}\right)^{-1}} \min \left\{u_{0} \sqrt{1+x^{2}}, \frac{1}{y}\left(\frac{1}{d h n}-\sqrt{1+x^{2}}\right)\right\} .
\end{aligned}
$$

Заменяя сумму по $n$ интегралом и вводя новую переменную интегрирования $r=n d h \sqrt{1+x^{2}}$, получаем

$$
\begin{aligned}
& \sum_{n \leqslant(d h)-1} \int_{0}^{1} \int_{0}^{1} \frac{\Psi_{0}(n d ; x, y)}{n} d x d y \\
& \quad=\int_{0}^{\alpha_{0}} \frac{d x}{1+x^{2}} \int_{0}^{t_{0}} d r \int_{0}^{1} d y \min \left\{u_{0}, \frac{1}{y}\left(\frac{1}{r}-1\right)\right\}+O(d h) \\
& =\frac{\pi^{3}}{3} \int_{0}^{\alpha_{0}} \frac{d x}{1+x^{2}} \int_{0}^{t_{0}} d r \int_{0}^{u_{0}} \rho(r, u) d u+O(d h) .
\end{aligned}
$$

Подстановка последней формулы в (16) дает

$$
\begin{aligned}
\widetilde{\Phi}_{ \pm}(h) & =\frac{\pi^{2}}{6} \int_{0}^{\alpha_{0}} \frac{d x}{1+x^{2}} \int_{0}^{t_{0}} d r \int_{0}^{u_{0}} \rho(r, u) d u \cdot \sum_{d \leqslant h^{-1}} \frac{\mu(d)}{d^{2}}+O_{\varepsilon}\left(h^{1 / 2-\varepsilon}\right)= \\
& =\int_{0}^{\alpha_{0}} \frac{d x}{1+x^{2}} \int_{0}^{t_{0}} d r \int_{0}^{u_{0}} \rho(r, u) d u+O_{\varepsilon}\left(h^{1 / 2-\varepsilon}\right) .
\end{aligned}
$$

Теорема, сформулированная во введении, полностью доказана. 


\section{ЛитеРАТУРА}

[1] М. О. Авдеева, О статистиках неполных частных конечных иепных дробей, Функц. анализ и его прил., 38:2 (2004), 1-11.

[2] Г. Полиа, Г. Сеге, Задачи и теоремы из анализа, т. 2, Наука, М., 1978.

[3] А. Я. Хинчин, Цепнъе дроби, Наука, М., 1978.

[4] F. P. Boca, R. N. Gologan, A. Zaharescu, The statistics of the trajectory of a certain billiard in a flat two-torus, Comm. Math. Phys., 240:1-2 (2003), 53-73.

[5] T. Estermann, On Kloosterman's sum, Mathematika, 8 (1961), 83-86.

Хабаровское отделение Института прикладной математики ДВО РАН e-mail: vab@iam.khv.ru

Хабаровское отделение Института прикладной математики ДВО РАН e-mail: ustinov@iam.khv.ru
Поступило в редакцию 24 января 2007 г. 